Pontifícia Universidade Católica $_{\text {a }}$

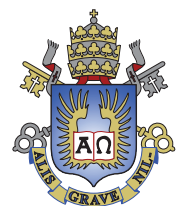

Carla Verônica Teixeira Sobrinho

\title{
Ruína e Resseguro: modelos contínuos e suas aproximações
}

\section{Dissertação de Mestrado}

Dissertação apresentada como requisito parcial para obtenção do grau de Mestre pelo Programa de Pós-graduação em Matemática do Departamento de Matemática da PUC-Rio

Orientador : Prof. Hélio Côrtes Vieira Lopes Co-Orientador: Prof. Álvaro de Lima de Veiga Filho 


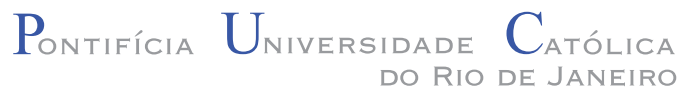

\title{
Carla Verônica Teixeira Sobrinho
}

\section{Ruína e Resseguro: modelos contínuos e suas aproximações}

Dissertação apresentada como requisito parcial para obtenção do grau de Mestre pelo Programa de Pós-graduação em Matemática do Departamento de Matemática do Centro Técnico Científico da PUC-Rio. Aprovada pela comissão examinadora abaixo assinada.

\author{
Prof. Hélio Côrtes Vieira Lopes \\ Orientador \\ Departamento de Matemática - PUC-Rio
}

Prof. Álvaro de Lima de Veiga Filho

Co-Orientador

Departamento de Engenharia Elétrica - PUC-Rio

Prof. Geovan Tavares dos Santos

Departamento de Matemática - PUC-Rio

Prof. Cristiano Augusto Coelho Fernandes Departamento de Engenharia Elétrica - PUC-Rio

Prof. Marcos Craizer Departamento de Matemática - PUC-Rio

Prof. José Eugênio Leal Coordenador do Centro Técnico Científico - PUC-Rio

Rio de Janeiro, 9 de Outubro de 2009 
Todos os direitos reservados. Proibida a reprodução total ou parcial do trabalho sem autorização da universidade, do autor e do orientador.

\section{Carla Verônica Teixeira Sobrinho}

Mestrado: Matemática - Pontifícia Universidade Católica do Rio de Janeiro - PUC-Rio (2007-2009).

Graduação: Licenciatura em Matemática - Universidade do Estado do Rio de Janeiro - UERJ (2001/2-2005).

Ficha Catalográfica

Sobrinho, Carla Verônica T.

Ruína e Resseguro: modelos contínuos e suas aproximações / Carla Verônica Teixeira Sobrinho; orientador: Hélio Côrtes Vieira Lopes; co-orientador: Álvaro de Lima de Veiga Filho. - Rio de Janeiro : PUC-Rio, Departamento de Matemática, 2009.

v., 71 f: il. ; $29,7 \mathrm{~cm}$

1. Dissertação (Mestrado em Matemática) - Pontifícia Universidade Católica do Rio de Janeiro, Departamento de Matemática.

Inclui referências bibliográficas.

1. Matemática - Tese. 2. Teoria da Ruína. 3. Modelo Clássico de Risco Coletivo. 4. Coeficiente de Ajuste. 5. Probabilidade de Ruína. 6. Resseguro. I. Côrtes Vieira Lopes, Hélio. II. Lima de Veiga Filho, Álvaro. III. Pontifícia Universidade Católica do Rio de Janeiro. Departamento de Matemática. IV. Título.

CDD: 510 


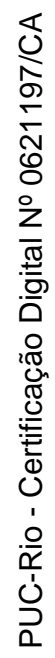

À minha mãe Izabel e ao meu marido Alexandre. 


\section{Agradecimentos}

Primeiramente, agradeço a Deus por ter me proporcionado e capacitado para vencer mais um desafio e sempre ter me dado forças nos momentos mais difíceis.

À minha mãe pelo esforço, dedicação e empenho na minha formação pessoal, profissional e espiritual.

Ao meu marido Alexandre, pelo incentivo, paciência, amor e carinho que ele me ofereceu durante este período.

Ao Professor Hélio Lopes, pela orientação, pela oportuidade concedida e por toda atenção e tempo dedicados ao desenvolvimento desta dissertação.

À minha família, pela compreensão da minha ausência durante este período.

Aos professores do programa de Pós-graduação em Matemática da Pontifícia Universidade Católica do Rio de Janeiro, pela contribuição na minha formação.

Às amigas Cleide, Joana e Marina, pela ajuda e participação em momentos importantes.

Aos amigos do mestrado, pela atenção e ajuda nos momentos necessários.

Aos amigos pessoais, pelo incentivo e apoio.

Aos membros da banca, por terem aceito o convite.

Aos funcionários da secretaria, pelo apoio durante este período. 


\section{Resumo}

Sobrinho, Carla Verônica T.; Côrtes Vieira Lopes, Hélio; Lima de Veiga Filho, Álvaro. Ruína e Resseguro: modelos contínuos e suas aproximações. Rio de Janeiro, 2009. 71p. Dissertação de Mestrado - Departamento de Matemática, Pontifícia Universidade Católica do Rio de Janeiro.

Nesta dissertação vamos estudar a teoria da ruína considerando o modelo clássico de risco coletivo desenvolvido por Cramér e Lundberg, no qual o número de indenizações que ocorrem até um período de tempo $t$ é modelado por um processo de Poisson homogêneo. Podemos dizer que uma seguradora está em ruína se sua reserva ficar negativa em algum instante t. A probabilidade deste evento ocorrer é chamada de probabilidade de ruína. Devido à dificuldade de encontramos uma fórmula fechada para a probabilidade de ruína eventual de uma seguradora, apresentaremos alguns estimadores numéricos que constam na literatura para a probabilidade de ruína eventual. Também vamos mostrar nesse trabalho como um contrato de resseguro pode influenciar na probabilidade de ruína eventual de uma seguradora. A eficácia dos estimadores da probabilidade de ruína eventual é verificada com dados simulados, onde são assumidos diferentes modelos probabilísticos para as indenizações.

\section{Palavras-chave}

Teoria da Ruína, Modelo Clássico de Risco Coletivo, Coeficiente de Ajuste, Probabilidade de Ruína, Resseguro. 


\section{Abstract}

Sobrinho, Carla Verônica T.; Côrtes Vieira Lopes, Hélio; Lima de Veiga Filho, Álvaro. Ruin and Reinsurance: continuous models and its approaches. Rio de Janeiro, 2009. 71p. MsC Thesis — Departament of Mathematics, Pontifícia Universidade Católica do Rio de Janeiro.

In this dissertation we discuss the Ruin Theory, considering the classic collective risk model developed by Cramér and Lundberg, in which the number of claims that occur until a period of time $t$ is modeled by a homogeneous Poisson process. We can say that an insurance company is in ruin if its reserve became negative at some instant $t$. The probability of this event to occur is called the ruin probability. Since it is difficult to find an explicit expression for the ruin probability of an insurance company, we present some numerical approaches for its estimation that are available in the literature. Also we show in this work how a reinsurance contract can alter the insurance company's ruin probability. The effectiveness of the estimators of the ruin probabilities is verified with simulated data, where it is assumed different probabilist models for the claims.

\section{Keywords}

Ruin Theory, Classic Collective Risk Model, Adjust Coefficient, Probability of Ruin, Reinsurance. 


\section{Sumário}

1 Introdução 11

2 Modelo Clássico de Cramér-Lundberg $\quad 14$

2.1 Conceitos fundamentais 14

2.2 Descrição do Modelo Clássico de Cramér-Lundberg 15

2.3 Probabilidade de Ruína 20

3 O limite superior de Lundberg para a probabilidade de ruína eventual $\quad 22$

3.1 Coeficiente de ajuste 22

3.2 Desigualdade de Lundberg 24

4 Expressão fechada para a probabilidade de ruína eventual quando as indenizações individuais são exponencialmente distribuídas $\quad 28$

5 Fórmula de Pollaczek-Khinchin $\quad 35$

5.1 Perda agregada máxima 35

5.2 A Fórmula de Pollaczek-Khinchin 37

6 Aproximações analíticas para o cálculo da probabilidade de ruína eventual 40

7 Aproximações numéricas para o cálculo da probabilidade de ruína eventual 42

7.1 Fórmula Recursiva de Panjer 42

7.2 Método dos limites inferior e superior para a probabilidade de ruína eventual 44

7.3 Método para a proximação da probabilidade de ruína eventual proposto por Dickson and Waters(1991) 46

$\begin{array}{ll}7.4 & \text { Exemplos numéricos }\end{array}$

$8 \quad$ Resseguro $\quad 54$

8.1 Resseguro e Coeficiente de Ajuste $\quad 57$

8.2 Resseguro e sua influência na probabilidade de ruína 62

$\begin{array}{lll}9 & \text { Conclusões e trabalhos futuros } & 68\end{array}$

$\begin{array}{ll}\text { Referências Bibliográficas } & 69\end{array}$ 


\section{Lista de figuras}

2.1 Exemplo de uma trajetória, obtida por simulação, de um processo de reserva $U(t)$, onde $X_{i} \sim \operatorname{Exp}(1), \lambda=1, c=1.3 e$ $u=10$.

2.2 Probabilidade de ruína em função da reserva inicial u.

3.1 Função de ajuste onde os temos como parâmetros do Processo de reservas $\lambda=30$ e $\theta=0.2$ e as indenizações individuais com distribuição $\operatorname{Gama}(2,0.01)$.

3.2 Probabilidade de ruína em função do tempo e o Limitante de Lundberg para um processo de parâmetros $\lambda=1, \theta=0.1 e$ indenizações individuais com distribuição Exponencial(0.1).

7.1 Discretização da distribuição de uma variável $X$.

7.2 Aproximações para a probabilidade de ruína eventual, para um modelo de risco coletivo onde as indenizações individuais tem distribuição exponencial com média 1.

7.3 Aproximações para a probabilidade de ruína eventual, para um modelo de risco coletivo onde as indenizações individuais tem distribuição gama com média 1.

7.4 Aproximações para a probabilidade de ruína eventual, para um modelo de risco coletivo onde as indenizações individuais tem distribuição pareto com média 1.

8.1 Exemplo do impacto de um contrato de resseguro proporcional em um processo de reservas $U(t)$ de uma seguradora.

8.2 Exemplo do impacto de um contrato de resseguro do tipo excesso de danos em um processo de reservas $U(t)$ de uma seguradora.

8.3 Função coeficiente de ajustes para os seguintes valores de $\xi$ : $\xi=0.25, \xi=0.3, \xi=0.35, \xi=0.4$.

8.4 Função coeficiente de ajustes para os seguintes valores de $\xi$ : $\xi=0.2, \xi=0.25, \xi=0.35, \xi=0.5$.

8.5 Comparação entres os valores de valores $\psi(u), \psi(u ; \alpha), \psi(u, \alpha)_{2}$ e o limite de Lundperg, para um modelo de risco coletivo, onde as indenizações individuais tem distribuição exponencial com média 1.

8.6 Comparação entres os valores de $\psi(u), \psi(u, M)_{2}$ e o limite de Lundberg, para um modelo de risco coletivo. 


\section{Lista de tabelas}

7.1 Valores para a probabilidade de ruína eventual encontrados através da expressão fechada, das aproximações analíticas e numéricas, para um modelo de risco coletivo com os seguintes parâmetros: $X \sim \operatorname{Exp}(1), \lambda=1, \theta=0.2$.

7.2 Erros relativos de cada aproximação, quando comparadas com o valor encontrado pela expressão fechada para a probabilidade de ruína eventual.

7.3 Valores para a probabilidade de ruína eventual encontrados através das aproximações analíticas e numéricas, para um modelo de risco coletivo com os seguintes parâmetros: $X \sim$ $\operatorname{Gama}(2,2), \lambda=1, \theta=0.2$.

7.4 Comparação entre as aproximações para a probabilidade de ruina eventual, para um modelo de risco coletivos com os seguintes parâmetros: $X \sim \operatorname{Pareto}(2,1), \lambda=1, \theta=0.2$.

8.1 Valores de $\alpha$ que maximizam $R(\alpha)$ para $\theta=0.2$ e para diferentes valores de $\xi$.

8.2 Valores $M^{*}$ que maximizam $R(M)$ para $\theta=0.15$ e diferentes valores de $\xi$.

8.3 Valores de $\alpha$ que minimizam a função $\psi(u, \alpha)$, para diferentes valores de reserva inicial $u$.

8.4 Comparação entre os valores de $\psi(u), \psi(u ; \alpha), \psi(u, \alpha)_{2}$ e o limite de Lundberg, para um modelo de risco coletivo.

8.5 Comparação entre os valores de $\psi(u ; M)$ encontrados pela expressão fechada e os valores encontrados pela aproximação numérica, para um modelo de risco coletivo onde as indenizações individuais tem distribuição exponencial com média $1, \lambda=1$, $\theta=0.1, \xi=0.15$.

8.6 Comparação entre os valores $\psi(u), \psi(u, M)_{2}$ e do limite de Lundberg, para um modelo de risco coletivo com indenizações individuais exponencialmente distribuídas com média $1, \theta=0.2$ e $\lambda=1$, no qual foi feito um contrato de resseguro do tipo excesso de danos. 